\title{
Implications of the emergency department triage environment on triage practice for clients with a mental illness at triage in an Australian context
}

\author{
Marc Broadbent, RN, PhD ${ }^{\text {a,* }}$ \\ Lorna Moxham, MHN, PhD ${ }^{b}$ \\ Trudy Dwyer, RN, PhD ${ }^{\mathrm{C}}$
}

a School of Nursing and Midwifery, University of the Sunshine Coast, Qld, Australia

b School of Nursing, Midwifery and Indigenous Health, University of Wollongong, Wollongong, NSW, Australia

c School of Nursing and Midwifery, Central Queensland University, Rockhampton, Qld, Australia

Received 1 November 2012; received in revised form 22 November 2013; accepted 27 November 2013

\section{KEYWORDS \\ Triage; \\ Mental health; \\ Environment; \\ Emergency nursing; \\ Caring}

\begin{abstract}
Summary
Background: The practice environment of the emergency department (ED) refers to both the people and physical factors (architecture) in the environment in which health care is provided. ED triage practice environments are the very places where caring or the delivery of health care often begins. This paper examines the implications of the emergency department triage practice environment on the triage practice of nurses who triage clients with a mental illness. Methods: An observational ethnographic approach inclusive of participant observation, formal and informal semi structured interviews, examination of documents and the collation of field notes were the means of data collection. Data was analysed through constant comparison and theoretical coding.

Results: Nurses who work in ED triage are cognisant of environmental impacts as they undertake rapid client assessment and manage busy and noisy waiting rooms. The triage environment does influence the ED triage assessment and the management of clients who present seeking mental health care.
\end{abstract}

* Corresponding author at: University of the Sunshine Coast, ML 40 Locked Bag 4, Maroochydore DC, Qld 4558, Australia. Tel.: +61 07 54594549; fax: +61 0754565940.

E-mail addresses: mbroadbent@usc.edu.au (M. Broadbent), Imoxham@uow.edu.au (L. Moxham), t.dwyer@cqu.edu.au (T. Dwyer). 
Conclusions: Tensions arise when the architectural environment of an ED triage area affects client behaviour, the capacity to provide optimal client care and the ability to conduct a triage assessment that obtains the best data possible. Understanding the impact of the ED triage practice environment on people with a mental illness facilitates an understanding of how people from this client group can be better supported in a complex and busy ED environment.

(c) 2013 College of Emergency Nursing Australasia Ltd. Published by Elsevier Ltd. All rights reserved.

\section{What is known}

Australian emergency departments (EDs) are the main portal for clients with a mental illness to access acute mental health care. In response to this there has been a move to improve mental health service provision to Australian EDS. However, the exact number of mental health presentations to Australian EDs is unknown. ED triage nurses find themselves having to care for clients with a mental illness, often in the ED waiting room until a $\mathrm{MH}$ clinician arrives. This poses challenges to the ED triage nurse.

\section{What this paper adds}

This paper describes the triage practice environment of an Australian ED and explores the issues associated with working in such an environment with a focus on assessment and management of clients with a mental illness. ED triage nurses try to create a therapeutic environment to demonstrate care for the client however the lack of appropriate, safe yet visible environments makes it difficult to manage some clients. It is posited that ED triage and waiting room environments should be the subject of further research to create environments that are safe, functional and provide therapeutic spaces for clients in need including those with a mental illness.

\section{Introduction}

There has been a constant increase in the number of clients who have a mental illness presenting to Australian emergency departments (EDs). This rise is largely attributed to effects associated with the mainstreaming of mental health services into general health services. ${ }^{1}$ There is evidence suggesting that EDs are not only experiencing increased presentations by clients with a mental illness but that they also present with increased acuity. ${ }^{2-4}$ Accurate statistics are not available to determine exactly how many clients with a mental illness access the ED. The most current reporting tool for emergency healthcare in Australia, the Australian Hospital Statistics 2009-10: Emergency Department Care and Elective Surgery Waiting Times ${ }^{5}$ surprisingly do not report on this data. Evidence of the increased presentations though can be gleaned from mental health occasions of service for clients who have been given a principal mental health diagnosis. Using this measure, it was noted that across Australia in the 2007-2008 reporting period there was a rise of 4.1 percent in mental health related ED occasions of service compared to the previous year. 2007-2008 saw 258,500 mental health related ED occasions of service. ${ }^{6}$ However, as previously alluded to, accurate reporting is unlikely particularly as a result of no nationally consistent approach to data collection for this client group.

Given that the number of ED occasions of service for 2006-2007 was 7.1 million, ${ }^{7}$ an estimate of 258,500 mental health occasions of service represents four percent of all ED occasions of service, remembering that this number is likely to be greater due to the underreporting of presentations. Morphet et al. ${ }^{8}$ suggest that 5-10 percent of all presentations to Australian EDs are mental health presentations. In response to the escalating numbers of mental health presentations to Australian EDs, health services have responded by introducing specialist mental health triage scales to aid decision making ${ }^{9}$ and by better positioning specialist mental health clinicians within EDs. ${ }^{10}$

Central to this paper is the notion that, from the point of presentation through to the handover of the client to a mental health clinician, the client with a mental illness remains the responsibility of the ED triage nurse. This paper concerns itself with the implications of the ED triage environment on the triage practice of nurses who triage clients with a mental illness in an Australian regional ED. This ED services a population of some 180,000 people and is located approximately $75 \mathrm{~km}$ from an Australian capital city. In particular the paper focuses on the passage of time when the care of the client is with the ED triage nurse and the influence of the ED triage environment on triage assessment and care delivery. The paper does not report on the client's experience of the triage process. Clients were not observed or interviewed for the purposes of the study and any discussion of client behaviour is based on the authors' experiences and included to contextualise the discussion.

\section{Literature review}

The principal document guiding ED design in Australia ${ }^{11}$ identifies that a dedicated area for the assessment and stabilisation of clients with a mental illness should be provided. Tension around the management of clients with a mental illness, largely undertaken by non-mental health trained staff, arises when ED triage staff are unable to leave clients unsupervised in these dedicated areas. Consequently, unwell, often distressed and untreated clients remain in the public ED triage waiting areas until such time as they are seen by a mental health clinician for post ED triage assessment. The management of clients in the triage waiting room environment is becoming an increasingly important area of discourse and one that is subject to growing practice and service delivery evaluation. 
Within the Australian context, ED triage area design is specifically detailed in two documents, the Policy on the Australasian Triage Scale ${ }^{12}$ and the Position Statement Triage Nurse. ${ }^{13}$ These documents identify a number of environmental considerations in relation to ED triage design and are applicable to all Australian EDs regardless of size and locale. The Australasian College of Emergency Medicine ${ }^{12}$ states that triage:

'...must be immediately accessible and clearly signposted. Its size and design must allow for patient examination, privacy and visual access to the entrance and waiting areas, as well as for staff security' (p. 2).

The word must in this context is of note as it implies the elements outlined are mandatory. Interestingly, the document also identifies that there should be a range of facilities available within a triage area. Such equipment is deemed to include emergency equipment, telephones and duress alarms. Should in this instance can be interpreted as desirable but not mandatory. The other policy document that dictates ED triage design, from the College of Emergency Nursing Australasia, ${ }^{13}$ lists six points necessary for ED triage design. The importance of the first five is also demonstrated by being prefixed with the word must. The five provisions are
1. accessibility
2. access to examination areas
3. provision of equipment
4. infection control measures and
5. be safe for staff and clients

The sixth consideration, like those within the aforementioned design suggestion, is prefixed by the word should, and relates to the environment facilitating privacy for clients. ${ }^{13}$ The fact that privacy concerns are documented as should, and not must is surprising, given the centrality of privacy and confidentiality to nursing practice. ${ }^{14}$ Despite the importance of the triage role to the function, efficacy, management and workload of the ED, there remains a paucity of literature that critiques the architectural environment of triage and waiting areas in emergency departments and the impact it has on the process of ED triage. What little literature there is, generally concerns itself with redesign and occupancy of new EDs ${ }^{15,16}$ or the redesign of emergency departments to improve client flow and ED processes. ${ }^{17}$

Literature does exist that explores the use of space in the context of care in both the ED and mental health settings. Nugus and Forero ${ }^{18}$ conducted an ethnographic study in an Australian ED and found that ED nurses' response to external pressures were organisationally driven and that the provision of quality care was balanced with the need to maintain operational efficiency. This may shed some light on the difficulty experienced by ED triage nurses managing clients with a mental illness. Clients with impaired insight may be disruptive, and therefore not be able to be managed for optimal organisational flow in the triage area.

Andes and Shattell ${ }^{19}$ further expanded on this assertion in their paper on the meanings of space and place in acute psychiatric care. The authors identify that the mental health status of a client is within the realm of all nurses' work. However, the responsibility to engage with clients may be overshadowed by more concrete operational tasks such as paperwork. They argue that the nursing station, or in the ED context, the triage desk, may pose an existential dilemma where nurses want to distance themselves, in order to get the job done, yet relate to clients at the same.

This tension is evidenced in triage nursing practice where triage nurses have responsibilities that reach far beyond the actual triage assessment of clients. Not only do they undertake rapid clinical assessments, they provide ongoing care to clients and their families or carers who are in the waiting room. The triage desk can be considered to be the epicentre of the triage area; a location of high people traffic, highly visible, very noisy and the locus of control of the triage area and waiting room. As the client often remains the responsibility of the ED triage nurse from the point of presentation, through to the handover of the client to the mental health clinician, the ED triage environment may influence the assessment and care of the client. The aim of this study is to explore the issues surrounding how ED triage nurses assess and manage clients with a mental illness in this complex, public environment.

\section{Research design}

The research used an ethnographic design to observe and understand emergency nurse's practices when triaging clients with a mental illness and the impact of the emergency department triage environment on their service delivery. Ethnography, with its emphasis on understanding the cultural influences of clinical practice within the practice environment was ideally suited to investigate emergency nursing practice as this methodology adds value to further understanding the ED world. ${ }^{20}$ This paper draws on research findings from a larger observational ethnographic study that examined the interdisciplinary relationships between the ED triage nurse and the specialist mental health triage nurse. Specifically, this paper concerns itself with one element of the broader study, ED triage practice environment and the effect of this on ED triage nurse practice.

\section{Setting}

The research setting was a hospital in regional Australia that had a large emergency department with in excess of 44,000 client separations per year. About four and a half percent of these client presentations are people requiring a mental health assessment.

\section{Participants}

The study ED had 45 staff qualified to conduct triage during the data collection period. Typically one emergency nurse was rostered to triage for the morning shift, two for the afternoon shift and one triage nurse for the night shift.

\section{Methods}

Consistent with the methodology, eight weeks was spent in the field gathering data. Data was collected using participant observation, formal and informal semi structured 
interviews with triage nurses, examination of documents and the collation of field notes. ${ }^{21}$ Participant observation, central to ethnographic research ${ }^{22}$ with observer as participant best reflected the position adopted for this research. In this role the researcher has face-to-face interaction with the participants in the setting with the main purpose of the interaction being to gather data. ${ }^{23}$ Interviews were conducted with a purposive sample of twenty-eight ED triage nurses all of whom had received triage training and were rostered to work at triage. At no point were clients presenting to triage interviewed. Observations that were made of the nurse-client interaction were limited to the effect of the triage practice environment on the ED triage nurses practice. Fetterman ${ }^{22}$ states that the interview affords the ethnographer the opportunity to contextualise what they see and hear and, as such, are the ethnographer's most important tool for data collection. Interviews allowed the researcher to confirm records of observations made in the field, and added to the iterative process of gaining deeper understanding of the issues under investigation. In total, two individual and ten group interviews were conducted with the ED triage nurse participants. The number of participants in an interview was dependent on the number of participants rostered on shift and available at the time the interviews were conducted. Interviews were taped recorded and transcribed verbatim prior to analysis.

\section{Data analysis}

A comprehensive and systematic approach was used to analyse the data. Thompson ${ }^{24}$ describes this as an eclectic process that is used for narrative review accompanied by ideas and intuitions, developed and documented during the fieldwork. Extensive notes from the taped interviews and from observations made by the researcher were used so as to articulate data into categories of meaning understood by the researcher.

The constant comparative method, as described by Glaser and Strauss ${ }^{25}$ in their seminal work on strategies for qualitative research, was used to analyse data generated during fieldwork. This systematic inductively driven method involved analysing individual elements of the data and comparing them to all other pieces of data through constant comparison and theoretical coding was undertaking by the authors independently of one another and then compared for consistency. Meaning drawn from the development of concepts and categories were explored through the examination of relevant literature.

\section{Ethics}

In order to undertake the research in a manner consistent with contempory ethical standards, the four pillars of ethical research - respect, research merit and integrity, justice and beneficence ${ }^{26}$ informed all stages of the research. All emergency triage nurses at the study site were given a plain English information sheet inviting them to participate in the study. Nurses agreeing to participate completed and returned a signed consent sheet. Participating nurses were informed of the voluntary and confidential nature of participation and of their right to withdraw without penalty. Prior to the commencement of the study ethical approval was gained from two appropriately constituted human research ethics committees; 08/35 and $\mathrm{H}$ 08/02-10.

\section{Findings}

\section{The triage environment}

Facing the front doors of the department in full view of the waiting room. The work space consists of two workspaces for the triage nurses and two workspaces for the ED triage clerks on one continuous desk. Each of these workspaces is equipped with a computer, document storage, filing cabinets and draws. A sheet of glass extended from the surface of these workspaces to the ceiling and across the full length of the triage desk. Participants explained the glass barrier had been installed as a security measure. At each of the four workspaces along the desk where a triage nurse or clerk is situated there is a $30-\mathrm{cm}$ wide gap in this glass wall. This small gap runs vertically to the ceiling positioned in the centre of the desk and it is through this small gap in the glass that communication between the triage nurse or clerk and the client occurs. Behind the triage desk, are filing cabinets filled with client notes, a clerical storage area and curtained cubicles with trolleys for clients who need to lie down prior to being moved into the main assessment area of the ED.

Throughout the triage area vinyl flooring and non-sound attenuating ceiling tiles contribute to what can be described as a very noisy environment. During times of high activity staff were observed speaking quite loudly in order to overcome local noise. This necessary increase in vocal projection makes the area even noisier with staff and clients having to speak louder and louder in order to make them heard and understood.

1600hrs - ED triage - large area - noisy - no sound attenuating tiles in the roof that means noise escalates easily (Researchers field notes).

This acoustic phenomenon is known as 'noise creep'27 and decreases the ability to have a private conversation despite many personal details being gathered by both the clerk and/or nurse at this point.

Numerous fluorescent lights that make the area very bright, stark and clinical in appearance also light the triage area. Access to the rear of the triage desk is only available to ED and other hospital staff as well as police and ambulance personal. The triage area is then, very much a public space with the triage nurse having no control over the movement of staff in and out of this initial assessment space. The triage nurses indicated they have no control over the ebb and flow of clients who present for care at the ED and those clients and family in the waiting room. This all contributed to a busy, noisy and non-private assessment area.

There is one main triage workspace that is exclusively allocated for use by the ED triage nurses at all times. During times of peak activity, typically during the afternoon, a second ED triage nurse may be deployed to the triage area and this nurse uses a separate workspace so that the triage nurses can work independently, but be seated next to each other. Both triage desks have a chair for clients requiring triage assessment who sit facing the triage nurse. Clients are triaged by the ED triage nurse then asked to speak to the clerk to complete admission paperwork, or bought directly into the ED for care if required. There are no walls or other 
barriers surrounding the client. On the staff side of the main triage desk there is a small stool positioned to the left hand side of the triage nurse. This stool, in close proximity to the triage area, may be used by various personnel waiting to speak to the triage nurse, regardless of whether there is a client providing information to the triage nurse or not. Anyone sitting on this stool can clearly hear what is being discussed at the point of triage, which further contributes to a lack of privacy.

Nurses who work in ED triage are cognisant of environmental impacts as they undertake their rapid client assessment and manage busy and noisy waiting rooms. Within these environments many different clients are seen and one such group, people who present with mental health problems, pose particular challenges for ED triage nurses. This picture of the triage practice setting provides an environmental contextualisation in which the following constructs of triage assessment and privacy can be understood.

\section{The triage assessment}

The practice environment has a direct influence on the ED triage nurses in regards to their ability to conduct an accurate and timely triage assessment particularly for clients who had a mental illness, inhibiting the collection of triage data:

\section{"It makes it very hard to make an assessment in two or three minutes when you can't extract the information that they'd give you if you were in a room just with one more other person than them. Which makes it very hard for us to give them the appropriate mental health triage category. I think that's probably our biggest issue, is how we triage them because of the facilities that we've got." (ED nurse)}

As described the ED triage area is an open public space that places considerable constraints on the ability to gather the information that is necessary for informed ED triage decision-making. ED triage nurses made it overtly clear that the lack of privacy was not just isolated to the fact that the client may be in a waiting room full of people. The following ED triage nurse was sensitive to the fact that privacy was lacking in the ED triage workspace:

"Even within our side of the wall. The lack of privacy for that patient..." (ED nurse)

The ED triage area is a place where staff like wardsmen, clerks and ambulance officers often gather. Large amounts of staff gathering in one area can be problematic. The following field note demonstrates this issue and reinforces the previous participants claim:

I'd hate to hear people speaking about me, there are 'multiple pairs of ears' - ambo, ward clerks, cleaners - [and this] compromises the transfer of information. (Researchers field note)

ED triage nurse participants expressed concern about the impact that the triage assessment has on the client and how sensitive they needed to be, particularly due to the lack of privacy in regards to maintaining confidentiality.
The following participant quote ratifies the previous researcher observation and illuminates the issue of how the ED practice environment can hinder effective triage assessment.

"Which is hard when there's no confidentiality at triage. At our triage there's nothing ... for anybody. So that makes it really hard for someone to open up and hard sometimes for you to listen, because this person's, you know, trying to give you some, you know, confidential and personal information and there's people standing right behind them. So yeah, that's why l'd like to be able to take them away and have a private conversation with them." (ED nurse)

That the triage area was noted to lack any opportunities for privacy during the initial triage process indicating that triage design may be predicated on factors that put safety and functionality above the need for privacy. Yet the principal function of triage is to obtain sometimes deeply personal and private information rapidly. In order to do this privacy needs to be considered equally with that of functionality and safety in order to facilitate triage decision-making.

\section{Client management}

The participants in this study indicated how the lack of opportunities to provide privacy had an impact on how they perceived clients could be managed.

Emergency department waiting rooms are places that clients who have been triaged as having low acuity illness or injury sit and pass the time, often for hours. The architectural design of the waiting room in this study contributed to it being noisy and had many distractions. Participants considered this to be a less than optimal environment for any unwell person, but particularly so for clients with a mental illness. One ED triage nurse participant commented:

\footnotetext{
"I don't think anyone with a mental health issue though is well cared for in a big waiting room with lots of people in it, where you're talking to them through glass, where you haven't got facilities where it is warm and cosy. It's a very open area, with everyone in their eyes ..." (ED nurse)
}

Field observations identified that the ED triage nurses felt it was imperative to find a private space for this client group and the provision of a separate, quiet space was important. Such concerns were said to be exacerbated if police officers brought in clients.

\begin{abstract}
"Even if they're not settled it's a place where the police don't have to sit holding their hand with the general public in full view. And it's got no distractions either if they're a bit manic or so forth it's not going to give them and distractions. It's safe, they can't hurt themselves ... In the waiting room they abscond, other patients criticise them, security are yelling at them. It's not comforting and reassuring for the patient or their family." (ED nurse)
\end{abstract}

A practice environment that facilitates privacy and confidentiality was felt to be in the best interests of all clients. Participants alluded to a therapeutic landscape facilitating 
effective management of the dynamics of the waiting room. Providing a quiet and safe place for clients who may be distressed, exhibiting signs of agitation or who are in a situation that is best not observed by the general public, such as a client with a police escort, was seen by participants as caring.

\section{Discussion}

The triage and management of clients with a mental illness who had to wait in a large public area was considered a particular challenge for ED triage nurse participants for a number of reasons; privacy and security were at the fore. ED triage nurses thought that minimising public scrutiny of a person in mental distress was imperative. ED triage nurses identified the need for a secure, private place, yet one that could be visible by the ED triage nurse, as necessary and important. Strathmann and $\mathrm{Hay}^{28}$ argue that an ED triage nurse helping clients to feel calm and assured demonstrates that, although not yet seen by a doctor, the caring has commenced. The provision of a secure, private environment is regarded as an everyday part of nursing practice and in areas like emergency departments is seen as an essential skill. ${ }^{29}$ It has long been known that the environment in which care is delivered has a profound effect on client outcomes and behaviour, particularly clients who have a mental illness. ${ }^{30-32}$ Nurses are often seen as builders of therapeutic landscapes that are said to contribute to restoration, rejuvenation and wellbeing. ${ }^{29}$ The desire by ED triage nurses to 'build such a landscape' and find a space for clients with a mental illness in which to comfortably wait was driven by a desire for safety and privacy, not only for the client, but also for the remainder of the people in the waiting room. Morphet et al. ${ }^{8}$ also noted that clients with a mental illness discussed several ways for improving the ED triage environment, including having dedicated mental health triage area, improved communication between staff and clients regarding plans of care, using single rooms for clients who present with a mental illness.

The issues associated with effectively triaging and managing clients with a mental illness in the ED waiting room were perceived as exacerbated, as ED triage nurses could not provide a therapeutic environment. Environmental characteristics do affect client behaviour and outcomes and provision of a private, safe and quiet area to wait that is visible from the ED triage area needs to be considered in ED design. Architectural considerations do though need to be cognizant of the principles of visibility and surveillance; both of which are critically important to the ED triage process. ${ }^{33}$ Vulnerable and fragile clients, who are emotionally disturbed, possibly aggressive, agitated and may be exhibiting bizarre behaviours often remain in the waiting room in the absence of suitable alternative areas. ED triage nurses opinions and clinical practice is influenced by their professional need to ensure client safety as well as the pragmatic demands of managing a usually full and noisy waiting room.

The discussion above highlights how participants perceive the practice environment to impact on how clients with a mental illness are both triaged and managed in the ED waiting rooms. The nature of the work undertaken by ED triage nurses reflects their needs in respect of providing privacy and security in order to enhance the ability to conduct the best triage assessment as possible and enhance the care of clients with a mental illness and others while waiting to be seen.

\section{Limitations}

These findings are from a study conducted in an ED in regional Australia with a small number of ED triage nurses. The comments by the participants reflect the social, cultural and historical contexts of the study site at the time of the research. Therefore the findings should be viewed in the context of the research setting at the time the research was conducted and may not be transferable or applicable to all EDs.

\section{Conclusion}

ED triage nurses work in an environment where there are often urgent requirements to access equipment and human physical resources quickly in order to facilitate rapid decision-making and to manage increasingly busy waiting rooms. Tensions arise when the architectural environment of an ED triage area affects client behaviour, the capacity to provide optimal client care and the ability to conduct a triage assessment that obtains the best data possible. Recent studies highlight the manner in which the built environment in health care disrupts effective communication thereby increasing staff and client stress. ${ }^{34,35}$ This paper has identified that while privacy is a requirement of policy documents guiding Australian triage design, it is not necessarily effectively incorporated as a design feature into ED triage and waiting room environments. This has a negative influence on the ability to garner accurate information critical for ED triage decision-making and to provide effective ongoing management of the client with a mental illness. Understanding of the impact of the practice environment on particular client groups, as the above discussion has provided, facilitates an understanding of how people with a mental illness can be better supported in a complex and busy ED triage environment. It is recommended that further research be conducted on ED triage and waiting room design, the client's experience of the ED triage experience and a complimentary study involving users of ED triage and ED triage staff.

\section{Contributions}

$M B, L M$ and TD conceived the research. MB gained ethical approval from both HRECS. MB collected the data and wrote the dissertation from which this paper has been drawn. This paper was written by MB and edited by MB, LM and TD.

\section{Provenance and conflict of interest}

This paper uses data from the first authors $\mathrm{PhD}$. Professor Moxham was the principal supervisor of the first author, Associate Professor Dwyer was the Co supervisor. There are no actual or potential conflicts of interest financial, personal or 
other relationships with other people or organisations within three years of beginning this paper that could inappropriately influence, or be perceived to influence, this paper. This paper was not commissioned.

\section{Funding}

There was no funding source for this research other that that normally available as part of a research by higher degree program.

\section{References}

1. Shafiei T, Gaynor N, Farrell G. The characteristics, management and outcomes of people identified with mental health issues in an emergency department. J Psychiatr Ment Health Nurs 2011;18(1):9-16.

2. Wand T, Schaecken P. Consumer evaluation of a mental health liason nurse service in the emergency department. Contemp Nurse 2006;21(1):14-21.

3. Marynowski-Traczyk D, Moxham L, Broadbent M. A critical discussion of the concept of recovery for mental health consumers in the emergency department. Aust Emerg Nurs $J$ 2013;16(3):96-102.

4. Marynowski-Traczyk D, Broadbent M. What are the experiences of emergency department nurses in caring for clients with a mental illness in the emergency department? Aust Emerg Nurs J 2011;14(3):172-9.

5. Australian Institute of Health and Welfare. Australian hospital statistics 2009-10: emergency department care and elective surgery waiting times, cat. no. HSE 93. Canberra: AlHW; 2011.

6. Australian Institute of Health and Welfare. Mental health services in Australia 2007-08, cat. no. HSE 88. Canberra: AlHW; 2010.

7. Australian Institute of Health and Welfare. Australian hospital statistics 2007-08, cat. no. HSE 71. Canberra: AlHW; 2009.

8. Morphet J, Innes K, Munro I, O’Brien A, Gaskin C, Reed F, et al. Managing people with mental health presentations in emergency departments - a service exploration of the issues surrounding responsiveness from a mental health care consumer and carer perspective. Aust Emerg Nurs J 2012;15(3):148-55.

9. Broadbent M, Moxham L, Dwyer T. Issues associated with the triage of clients with a mental illness in Australian emergency departments. Aust Emerg Nurs J 2010;13(4):117-23.

10. Wand $\mathrm{T}$, White $\mathrm{K}$. Examining models of mental health service delivery in the emergency department. Aust N Z J Psychiatry 2007; 41:784-91.

11. Australasian College of Emergency Medicine. G15 emergency department design. Melbourne: Australasian College of Emergency Medicine; 2007.

12. Australasian College for Emergency Medicine. PO6 policy on the Australasian triage scale. Melbourne: Australasian College for Emergency Medicine; 2006.

13. College of Emergency Nursing Australasia. Position statement - triage nurse. Melbourne: College of Emergency Nursing Australasia; 2009.

14. Nayeri N, Aghajani M. Patients' privacy and satisfaction in the emergency department: a descriptive analytical study. Nurs Ethics 2010;17(2):167-77.
15. Virtue E. Royal Melbourne Hospital emergency department redevelopment - the journey continues. Australas Emerg Nurs J 2009;17(7):168.

16. Flanagan $T$, Haas A. Planning a new emergency department: from design to occupancy. J Ambul Care Manage 2005;28(2):171-81.

17. Martin M, Champion R, Kinsman L, Masman K. Mapping patient flow in a regional emergency department: a model driven approach. Int Emerg Nurs 2011;19(2):75-85.

18. Nugus $P$, Forero R. Understanding interdepartmental and organisational work in the emergency department: an ethnographic approach. Int Emerg Nurs 2010;19:69-74.

19. Andes M, Shattell M. An exploration of the meanings of space and place in acute psychiatric care. Issues Ment Health Nurs 2006;27:699-707.

20. Cooper S, Endacott R, Chapman Y. Qualitative research: specific designs for qualitative research in emergency care. Emerg Med J 2009;26:773-6.

21. Roper S, Shapira J. Ethnography in nursing research. Thousand Oaks: Sage; 2000.

22. Fetterman D, Ethnography. In: Bickman L, Rog D, editors. Applied social research methods. 2nd ed. Thousand Oaks: Sage; 2009.

23. Ethnography participant observation. Russell C, Minichiello V, Sullivan G, Greenwood K, Axford R, editors. Research methods for nursing and health sciences. 2nd ed. Australia: Pearson Education; 2004.

24. Thomson D. The social meaning and function of humour in physiotherapy practice: an ethnography. Physiother Theory Pract 2010;26(1):1-11.

25. Glaser B, Strauss A. The discovery of grounded theory: strategies for qualitative research. Chicago: Aldine; 1967.

26. National Health and Medical Research Council \& Australian ViceChancellors Committee. National statement on ethical conduct in human research. Canberra: Australian Government; 2009.

27. Field C. Acoustic design in green buildings. ASHRAE J 2008;50(9):60-70.

28. Strathmann C, Hay MC. Working the waiting room: managing fear, hope, and rage at the clinic gate. Med Anthropol 2009;28(3):212-34.

29. Andrews G, Shaw D. Clinical geography: nursing practice and the (re) making of space. J Nurs Manag 2008;16:463-73.

30. Friis S. Characteristics of a good ward atmosphere. Acta Psychiatr Scand 1986;74:469-73.

31. Lanza M, Kayne H, Hicks C, Milner J. Environmental characteristics related to patient assault. Issues Ment Health Nurs 1994; 15:319-35.

32. Long CG, Langford V, Clay R, Craig L, Hollin RC. Architectural change and effects on the perceptions of the ward environment in a medium secure unit for women. $\mathrm{Br} J$ Forensic Pract 2011;13(3):205-12.

33. Commonwealth Department of Health and Ageing. Emergency triage education kit. Canberra: Australian Government; 2007.

34. Foureur M, Davis D, Fenwick J, Leap N, ledema R, Forbes I, et al. The relationship between birth unit design and a safe, satisfying birth: developing a hypothetical model. Midwifery 2010;26(5):520-5.

35. Olsen JC, Cutcliffe B, O’Brien BC. Emergency department design and patient perceptions of privacy and confidentiality. J Emerg Med 2008;35(3):317-20. 